\title{
Concurrent ARID1A and ARID1B inactivation in endometrial and ovarian dedifferentiated carcinomas
}

\author{
Mackenzie Coatham ${ }^{1}$, Xiaodong $\mathrm{Li}^{2}$, Anthony N Karnezis ${ }^{3}$, Lien N Hoang ${ }^{3,4}$, \\ Basile Tessier-Cloutier ${ }^{3}$, Bo Meng ${ }^{2}$, Robert A Soslow ${ }^{4}$, C Blake Gilks ${ }^{3}$, David G Huntsman ${ }^{3}$, \\ Colin J R Stewart ${ }^{5}$, Lynne M Postovit ${ }^{1}$, Martin Köbel ${ }^{6,7}$ and Cheng-Han Lee ${ }^{2,7}$ \\ ${ }^{1}$ Department of Oncology, University of Alberta, Edmonton, Alberta, Canada; ${ }^{2}$ Department of Laboratory \\ Medicine and Pathology, Royal Alexandra Hospital and University of Alberta, Edmonton, Alberta, Canada; \\ ${ }^{3}$ Department of Pathology and Laboratory Medicine, and Genetic Pathology Evaluation Center, Vancouver \\ General Hospital and University of British Columbia, Vancouver, British Columbia, Canada; ${ }^{4}$ Department of \\ Pathology, Memorial Sloan Kettering Cancer Center, New York, NY, USA; ${ }^{5}$ Department of Histopathology, \\ King Edward Memorial Hospital and School for Women's and Infants' Health, University of Western Australia, \\ Perth, Western Australia, Australia and ${ }^{6}$ Department of Pathology and Laboratory Medicine, Calgary \\ Laboratory Services and University of Calgary, Calgary, Alberta, Canada
}

\begin{abstract}
Dedifferentiated carcinoma of the endometrium or the ovary is an aggressive epithelial malignancy that comprises an endometrioid carcinoma together with an undifferentiated carcinoma. We recently reported that inactivation of BRG1 or INI1, core subunits of the switch/sucrose non-fermenting (SWI/SNF) complex, was the likely molecular event underlying dedifferentiation in about half of dedifferentiated carcinomas. In this study, we performed a genomic screen that included other members of the SWI/SNF complex to better delineate the molecular basis in the remainder of these tumours. We identified concurrent inactivating mutations involving $A R I D 1 A$ and $A R I D 1 B$ in 12 of 24 BRG1/INI1-intact, 0 of 3 INI1-deficient and 0 of 16 BRG1-deficient dedifferentiated carcinomas. All ARID1A and ARID1B co-mutated tumours displayed loss of ARID1A expression in the undifferentiated component with 11 of 12 tumours also displaying absent staining in the endometrioid component. ARID1B expression was absent in the undifferentiated component in all 12 tumours, whereas the corresponding endometrioid component showed intact expression. Clinically, ARID1A/ARID1B co-inactivated tumours showed similar aggressive behaviour to BRG1 or INI1-inactivated tumours. Given that ARID1A and ARID1B are the only known DNA-binding subunits of the SWI/SNF-A complex, additional inactivation of ARID1B in an ARID1A-deficient background appears to represent an alternative mechanism of disruption of SWI/SNFmediated transcriptional regulation, resulting in arrested cellular differentiation in endometrial and ovarian endometrioid cancer.
\end{abstract}

Modern Pathology (2016) 29, 1586-1593; doi:10.1038/modpathol.2016.156; published online 26 August 2016

Dedifferentiated carcinoma of the endometrium and the ovary refers to the occurrence of an endometrioid carcinoma juxtaposed to a histologically and immunophenotypically undifferentiated carcinoma. ${ }^{1,2}$ Recent molecular studies have demonstrated a

Correspondence: Dr C-H Lee, MD, PhD, Department of Laboratory Medicine and Pathology, Royal Alexandra Hospital and University of Alberta, Room DTC 5002-16, 10240 Kingsway, Edmonton, AB T5H 3V9, Canada.

E-mail: chenghanlee@gmail.com

${ }^{7}$ Co-senior authors.

Received 28 April 2016; revised 15 July 2016; accepted 18 July 2016; published online 26 August 2016 common clonal origin between the undifferentiated and differentiated (endometrioid) components in dedifferentiated carcinomas, indicating that these are not collision tumours but instead tumours that exhibit histologic progression presumably from the endometrioid component to the undifferentiated component. ${ }^{3,4}$ The presence of the undifferentiated carcinoma appears to confer an aggressive clinical course with most patients experiencing a progressive disease course and succumbing to the disease within a few years of initial diagnosis. This included cases where the undifferentiated carcinoma constitutes a minor proportion of the tumour. Moreover, in patients with higher stage disease, the foci of 
extrauterine spread are typically composed of undifferentiated carcinoma, ${ }^{5,6}$ indicating that it is the undifferentiated carcinoma component instead of the differentiated endometrioid carcinoma component that is behaving more aggressively.

We recently identified inactivating mutations of the SMARCA4 (encoding BRG1) or SMARCB1 (encoding INI1) genes, which encode for two ubiquitously expressed core subunits of the switch/ sucrose non-fermenting (SWI/SNF) chromatin remodelling complex, in the undifferentiated component of a subset of dedifferentiated endometrial carcinomas. $^{3,7,8}$ This results in loss of BRG1 or INI1 expression that was restricted to the undifferentiated tumour component, with the loss of BRG1 or INI1 expression being mutually exclusive. This was accompanied by the loss of PAX8 and oestrogen receptor (ER) in the undifferentiated component, indicating a loss of cellular differentiation due at least in part to disrupted SWI/SNF complex activity. ${ }^{9-12}$ However, inactivation of BRG1 or INI1 was only found in about half of histologically prototypical dedifferentiated endometrial carcinomas and the mechanism(s) underlying dedifferentiation in the remaining tumours remains undefined.

In this study, we performed targeted sequencing analysis on a larger number of SWI/SNF complex proteins in a series of endometrial and ovarian dedifferentiated carcinomas. We identified concurrent inactivating mutations in $A R I D 1 A$ and $A R I D 1 B$ that result in the loss of expression for these proteins in about a quarter of dedifferentiated carcinomas. These findings demonstrate the multitude of mechanisms for SWI/SNF complex inactivation that is involved in dedifferentiated endometrial/ovarian carcinomas.

\section{Materials and methods}

\section{Study Samples}

The study included 19 BRG1/INI1-intact dedifferentiated carcinomas and 24 BRG1/INI1-deficient dedifferentiated carcinomas (Table 1). $3,8,12$ These cases were obtained from Calgary Laboratory Services (Calgary, Canada), Royal Alexandra Hospital (Edmonton, Canada), Memorial Sloan Kettering Cancer Centre (New York, USA) and King Edward Memorial Hospital (Perth, Australia). All of the endometrial carcinomas included in this study were from hysterectomy specimens and all cases were centrally reviewed (MK, CHL and CJS), fulfilling the morphologic features described by Silva et al..$^{1,13}$ The study was approved by the Institutional Review Board (University of Alberta, Pro00042667).

\section{Targeted Gene Panel Sequencing Analysis and Validations}

For each case, tissue cores $(0.6 \mathrm{~mm})$ of tumour (with the endometrioid and the undifferentiated component separately cored whenever possible) and the corresponding normal tissues were obtained from formalin-fixed paraffin-embedded blocks. The tumour cores were obtained from areas showing the most histologically viable tumour with the least amount of stromal tissue. Normal tissue (ie, uterine cervix, fallopian tube and ovary) that was distant from and uninvolved by endometrial carcinoma was used for comparison. DNA was extracted from the tissue cores using the Qiagen formalin-fixed paraffinembedded tissue DNA extraction kit based on the manufacturer's protocols.

We designed a custom targeted sequencing panel (Illumina Truseq panel) using Illumina DesignStudio that covered an expanded list of the SWI/SNF complex proteins (ARID1A, ARID1B, ARID2, PBRM1, PHF10, SMARCA2, SMARCA4, SMARCB1, SMARCC1 and SMARCC2). The panel included 533 amplicons (175 bp) that covered 99\% of the exons and untranslated regions of these 10 genes. Custom amplicon libraries were prepared starting with $250 \mathrm{ng}$ of DNA as per Ilumina's Custom TruSeq Library Preparation protocol as previously described. ${ }^{14}$ Analysis was performed using the MiSeq Reporter and somatic variant caller 3.2.3.0. There was an average of 2195-fold coverage per amplicon and $95 \%$ of the amplicons had a median coverage in the 36 tumour samples of at least 50-fold. Only frameshift, nonsense and indel mutations (inactivating mutations) passing quality filter with at least $10 \%$ variant allele frequency were identified and orthogonally validated by direct Sanger sequencing. Further comparisons were made with the corresponding genomic sequences from normal tissue to determine the somatic/germline status of these mutations.

\section{Immunohistochemistry and Interpretation}

Immunohistochemistry was performed on whole tissue sections. The slides were incubated with antibodies to ARID1A (1:200, HPA005456, Sigma, Oakville, ON, Canada), ARID1B (1:100, clone 2D2, H00057492-M01, Abnova, Taipei, Taiwan), BRG1 (1:25, clone EPNCIR111A, ab110641, Abcam, Toronto, ON, Canada) and INI1 (1:50, 25/BAF47, 612110, BD Biosciences, Mississauga, ON, Canada); BRG1 and INI1 were processed using Ventana Benchmark XT (Ventana Medical Systems, Tucson, AZ, USA), whereas ARID1A and ARID1B were processed using Dako Omnis Autostainer (Dako Canada ULC, Mississauga, ON, Canada). The detection system used was the Bond polymer refine (Leica Microsystems, Wetzlar, Germany). For mismatch repair (MMR) proteins (MLH1, MSH2, MSH6 and PMS2), the primary antibodies used and the staining methods are the same as that reported previously. ${ }^{15,16}$ The slides were incubated with MLH1 (DAKO clone ES05 1:100), MSH2 (NCL clone 25D12 prediluted, Concord, ON, Canada), MSH6 
Table 1 Clinicopathologic features of 43 dedifferentiated carcinoma of the endometrium or the ovary

\begin{tabular}{|c|c|c|c|c|c|c|c|c|c|c|c|c|c|c|}
\hline \multirow{2}{*}{ Case number } & \multirow{2}{*}{ Tumour site } & \multirow{2}{*}{ Age } & \multirow{2}{*}{ FIGO stage } & \multicolumn{2}{|c|}{$B R G 1$} & \multicolumn{2}{|c|}{ INI1 } & \multicolumn{2}{|c|}{ ARID1A } & \multicolumn{2}{|c|}{$A R I D 1 B$} & \multirow{2}{*}{$M M R$} & \multirow{2}{*}{$\begin{array}{c}\text { Follow-up } \\
\text { duration (year) }\end{array}$} & \multirow{2}{*}{$\begin{array}{l}\text { Follow-up } \\
\text { status }\end{array}$} \\
\hline & & & & $E C$ & $U C$ & $E C$ & $U C$ & $E C$ & $U C$ & $E C$ & $U C$ & & & \\
\hline 1 & Uterus & 54 & II & + & - & + & + & + & + & + & + & Deficient & 0.75 & NED \\
\hline 2 & Uterus & 75 & IB & + & - & + & + & - & - & + & + & Deficient & 2.75 & NED \\
\hline 3 & Uterus & 54 & IA & + & - & + & + & - & - & + & + & Deficient & 2.62 & NED \\
\hline 4 & Uterus & 51 & IIIC2 & + & - & + & + & - & - & + & + & Deficient & 1.51 & DOD \\
\hline 5 & Uterus & 66 & IA & + & - & + & + & + & + & + & + & Deficient & 2.50 & NED \\
\hline 6 & Uterus & 69 & II & + & - & + & + & + & + & + & + & Intact & 0.61 & DOD \\
\hline 7 & Uterus & 70 & IIIC1 & + & - & + & + & - & - & + & + & Deficient & 1.17 & DOD \\
\hline 8 & Uterus & 73 & IA & + & - & + & + & + & + & + & + & Deficient & 1.17 & DOD \\
\hline 9 & Uterus & 66 & IB & + & - & + & + & - & - & + & + & Deficient & 4.00 & NED \\
\hline 10 & Uterus & 65 & IV & + & - & + & + & + & + & + & + & Intact & 0.13 & DOD \\
\hline 11 & Uterus & 55 & IV & + & - & + & + & - & - & + & + & Intact & 0.17 & DOD \\
\hline 12 & Uterus & 82 & IB & + & - & + & + & - & - & + & + & Deficient & 0.83 & DOD \\
\hline 13 & Uterus & 65 & IB & + & - & + & + & + & - & + & + & Deficient & 3.92 & NED \\
\hline 14 & Uterus & 59 & IIIA & + & - & + & + & + & + & + & + & Intact & 0.50 & DOD \\
\hline 15 & Ovary & 56 & IV & + & - & + & + & - & - & + & + & Intact & 0.33 & DOD \\
\hline 16 & Uterus & 54 & IIIC1 & + & - & + & + & + & + & + & + & Intact & 1.12 & DOD \\
\hline 17 & Uterus & 54 & IA & + & + & + & - & + & + & + & + & Intact & 1.25 & NED \\
\hline 18 & Uterus & 46 & IA & + & + & + & - & + & + & + & + & Intact & 4.13 & NED \\
\hline 19 & Uterus & 81 & IB & + & + & - & - & - & - & + & + & Deficient & 3.67 & NED \\
\hline 20 & Uterus & 56 & IA & + & + & + & + & + & - & + & - & Deficient & 0.25 & DOD \\
\hline 21 & Uterus & 57 & II & + & + & + & + & + & - & + & - & Deficient & 0.33 & NED \\
\hline 22 & Uterus & 57 & IA & + & + & + & + & - & - & + & - & Deficient & 1.34 & NED \\
\hline 23 & Uterus & 72 & IA & + & + & + & + & - & - & + & - & Deficient & 1.42 & NED \\
\hline 24 & Uterus & 63 & IIIC1 & + & + & + & + & - & - & + & - & Intact & 0.45 & DOD \\
\hline 25 & Uterus & 55 & IIIB & + & + & + & + & - & - & + & - & Deficient & 0.17 & DOD \\
\hline 26 & Uterus & 43 & IB & + & + & + & + & - & - & + & - & Deficient & 2.40 & NED \\
\hline 27 & Uterus & 81 & IB & + & + & + & + & - & - & + & - & Deficient & 0.25 & DOD \\
\hline 28 & Uterus & 71 & IA & + & + & + & + & - & - & + & - & Deficient & 0.92 & DOD \\
\hline 29 & Uterus & 52 & III & + & + & + & + & - & - & + & - & Intact & 0.08 & DOD \\
\hline 30 & Ovary & 56 & II & + & + & + & + & - & - & + & - & Deficient & 0.17 & DOD \\
\hline 31 & Ovary & 46 & II & + & + & + & + & - & - & + & - & Intact & 0.67 & DOD \\
\hline 32 & Uterus & 60 & II & + & + & + & + & - & - & + & + & Deficient & 6.37 & NED \\
\hline 33 & Uterus & 56 & IA & + & + & + & + & - & - & + & + & Intact & 0.42 & NED \\
\hline 34 & Uterus & 68 & IB & + & + & + & + & - & - & + & + & Deficient & 0.50 & NED \\
\hline 35 & Uterus & 93 & IB & + & + & + & + & + & + & + & + & Deficient & 0.40 & DOD \\
\hline 36 & Uterus & 73 & IIIA & + & + & + & + & + & + & + & + & Deficient & 0.86 & DOD \\
\hline 37 & Uterus & 60 & IB & + & + & + & + & + & + & + & + & Deficient & 1.83 & NED \\
\hline 38 & Uterus & 69 & IB & + & + & + & + & - & - & + & + & Deficient & NA & NA \\
\hline 39 & Uterus & 39 & IA & + & + & + & + & + & + & + & + & Intact & 2.00 & NED \\
\hline 40 & Uterus & 56 & IIIA & + & + & + & + & + & + & + & + & Intact & 3.41 & DOD \\
\hline 41 & Uterus & 73 & IB & + & + & + & + & + & + & + & + & Deficient & 3.75 & NED \\
\hline 42 & Uterus & 65 & III & + & + & + & + & - & - & + & + & Deficient & 4.75 & NED \\
\hline 43 & Uterus & 49 & III & + & + & + & + & + & + & + & + & Intact & NA & NA \\
\hline
\end{tabular}

Abbreviations: DOD, died of disease; EC, endometrioid component; MMR, mismatch repair protein status by immunohistochemistry; NA, information not available; NED, no evidence of disease; UC, undifferentiated component.

(BD Biosciences 44/MSH6 1:2000), PMS2 (BD Biosciences A16-4 1:100) and processed using the Leica Bond Max platform (Leica Microsystems) as per manufacturer's protocol with proprietary reagents. PAX8 immunohistochemistry (clone BC12, catalogue number: ACI 438, Biocare Medical, Concord, CA, USA) was performed as previously described. ${ }^{12}$ The differentiated and undifferentiated components were scored separately (MK and CHL). For ARID1A, ARID1B, BRG1, INI1 and MMR proteins, cases were scored as showing intact expression if any tumour cell nuclei showed nuclear staining and deficient if the tumour nuclei were unstained in the presence of internal positive control immunoreactivity. For PAX8, only nuclear staining was considered and was scored as positive if any tumour cells exhibited moderate to strong positive (definite) nuclear staining.

\section{Results}

\section{Study Series}

This study included 40 dedifferentiated endometrial carcinomas (18 BRG1 or INI1-deficient) that have been previously described and 3 dedifferentiated ovarian carcinomas (1 BRG1-deficient) (Table 1). For patients with dedifferentiated endometrial carcinoma, the age at the time of initial diagnosis ranged from 39 to 93 years with a median age of 62 years. Nearly a third of these patients (33\%) presented with 
extrauterine disease spread at presentation and nearly all of the patients with FIGO (The International Federation of Gynecology and Obstetrics) stages 3-4 disease died from the disease (typically within a year of the initial diagnosis). The three patients with dedifferentiated ovarian carcinoma ranged from 46 to 56 years in age; two patients presented with FIGO stage 2 diseases while one patient presented with FIGO stage 4 disease. All three patients experienced progressive disease and died from their disease within 8 months of disease diagnosis. The differentiated component of the 40 endometrial and 3 ovarian tumours were all endometrioid in type, being low-grade (FIGO grades 1 or 2 ) in 38 of 40 endometrial tumours and in 3 of 3 ovarian tumours. We have previously examined BRG1 and INI1 expression in this cohort of tumours by immunohistochemistry. ${ }^{3,8,12}$ As shown in Figure 1, 19 tumours (including 1 of the 3 ovarian tumours) showed absent BRG1 or INI1 expression in the undifferentiated component and intact BRG1 and INI1 expression in the corresponding endometrioid component, whereas 21 tumours showed intact BRG1 and INI1 expression in both the endometrioid and the undifferentiated components.

\section{Targeted Sequencing Analysis}

In the BRG1/INI1-deficient cohort $(n=19)$, we identified somatic SMARCA4 inactivating mutations (nonsense and/or frameshift) in 15 of 16 BRG1deficient tumours and somatic SMARCB1 inactivating mutations in 3 of 3 INI1-deficient tumours (Figure 1 and Supplementary Table 1). Inactivating mutations in
ARID1A were present in 12 cases. In the BRG1/INI1intact cohort $(n=24)$, inactivating somatic mutations in ARID1A and ARID1B were identified in 17 and 12 cases, respectively (Figure 1). For ARID1A and $A R I D 1 B, 58 \%$ and $50 \%$ of the inactivating mutations identified were frameshift mutations, whereas $42 \%$ and $50 \%$ were nonsense mutations, respectively. Concurrent ARID1A and ARID1B mutations were present in 12 of 24 BRG1/INI1-intact tumours (including 2 ovarian dedifferentiated carcinomas), whereas none of the BRG1-deficient or INI1-deficient tumours harboured concurrent ARID1A and ARID1B inactivating mutations (Figure 1). None of the cases harboured $A R I D 1 B$ inactivating mutations without concurrent ARID1A inactivating mutations.

We surveyed the COSMIC database for concurrent ARID1A and ARID1B inactivating (nonsense/frameshift/indel) mutations in endometrial and ovarian cancers and they were rare, present in only 3 of 631 endometrial carcinomas (all endometrioid in histotype) and 1 of 831 ovarian carcinomas (clear cell histotype). ${ }^{17}$ Given the frequent finding of concurrent $A R I D 1 A$ and $A R I D 1 B$ inactivating mutations in dedifferentiated carcinomas, particularly in the BRG1/INI1-intact cohort, we proceeded to evaluate the expression of ARID1A and ARID1B by immunohistochemistry.

\section{ARID1A and ARID1B Immunohistochemistry}

Among the 12 ARID1A/ARID1B co-mutated tumours, 10 showed absent ARID1A nuclear expression in both the endometrioid and the undifferentiated components, whereas 2 showed absent ARID1A

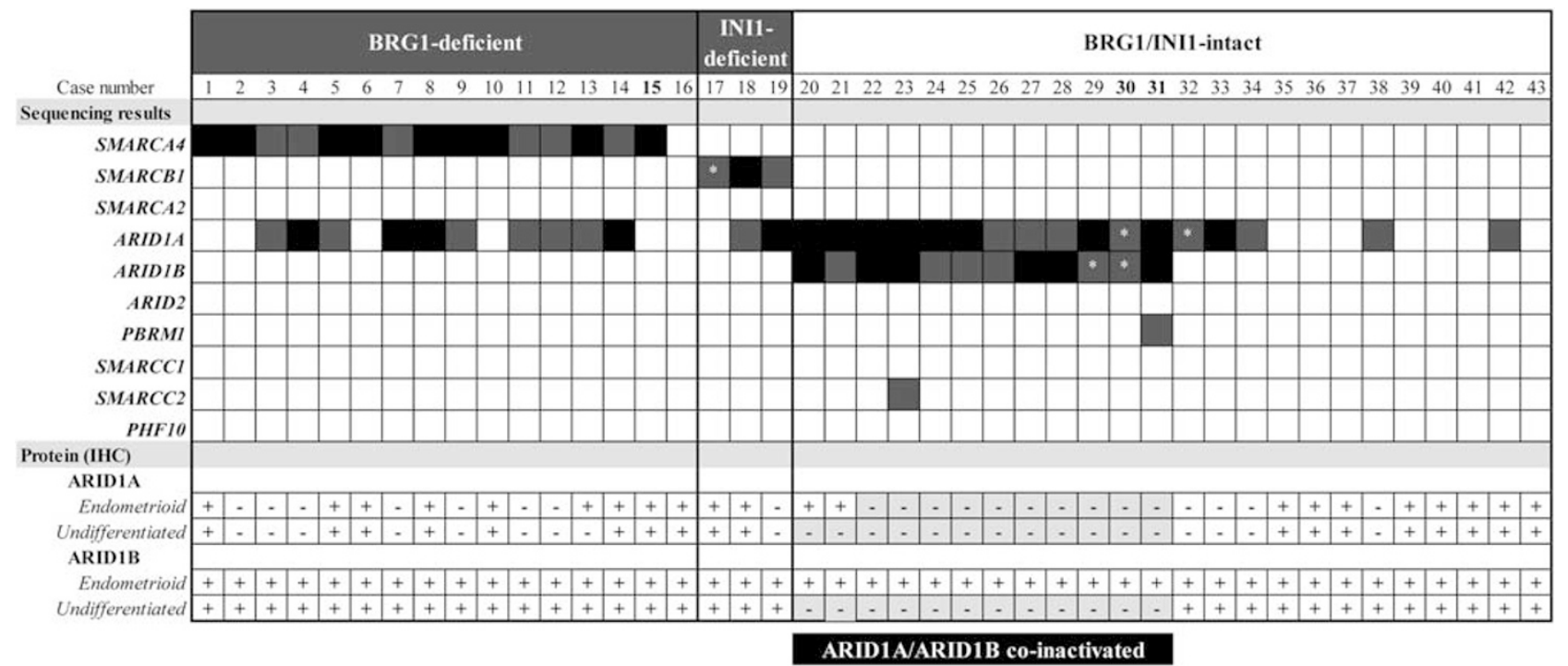

Figure 1 Concurrent ARID1A and ARID1B co-inactivation in a subset of dedifferentiated endometrial and ovarian carcinomas. Sequencing findings are summarized here and the heatmap depicts only inactivating mutations (frameshift and nonsense mutations) with dark grey colour indicating the presence of one inactivating mutation and black colour indicating the presence of two inactivating mutations. Mutations with $>70 \%$ frequency in sequenced reads (which are likely homozygous in nature) are further indicated by an asterisk. Cases in bold $(15,30$ and 31$)$ are ovarian tumours. Protein expression results are displayed with the endometrioid and the undifferentiated component shown separately; the presence of nuclear protein expression is indicated by $(+)$ with the absence of nuclear protein expression indicated by (-). IHC: immunohistochemistry. 
expression only in the undifferentiated component (Table 1 and Figure 2). ARID1B expression was intact in the endometrioid component but completely absent in the undifferentiated component of all 12 tumours (Figure 2). Nine of the 12 ARID1A/ARID1B co-deficient tumours also exhibited MMR protein
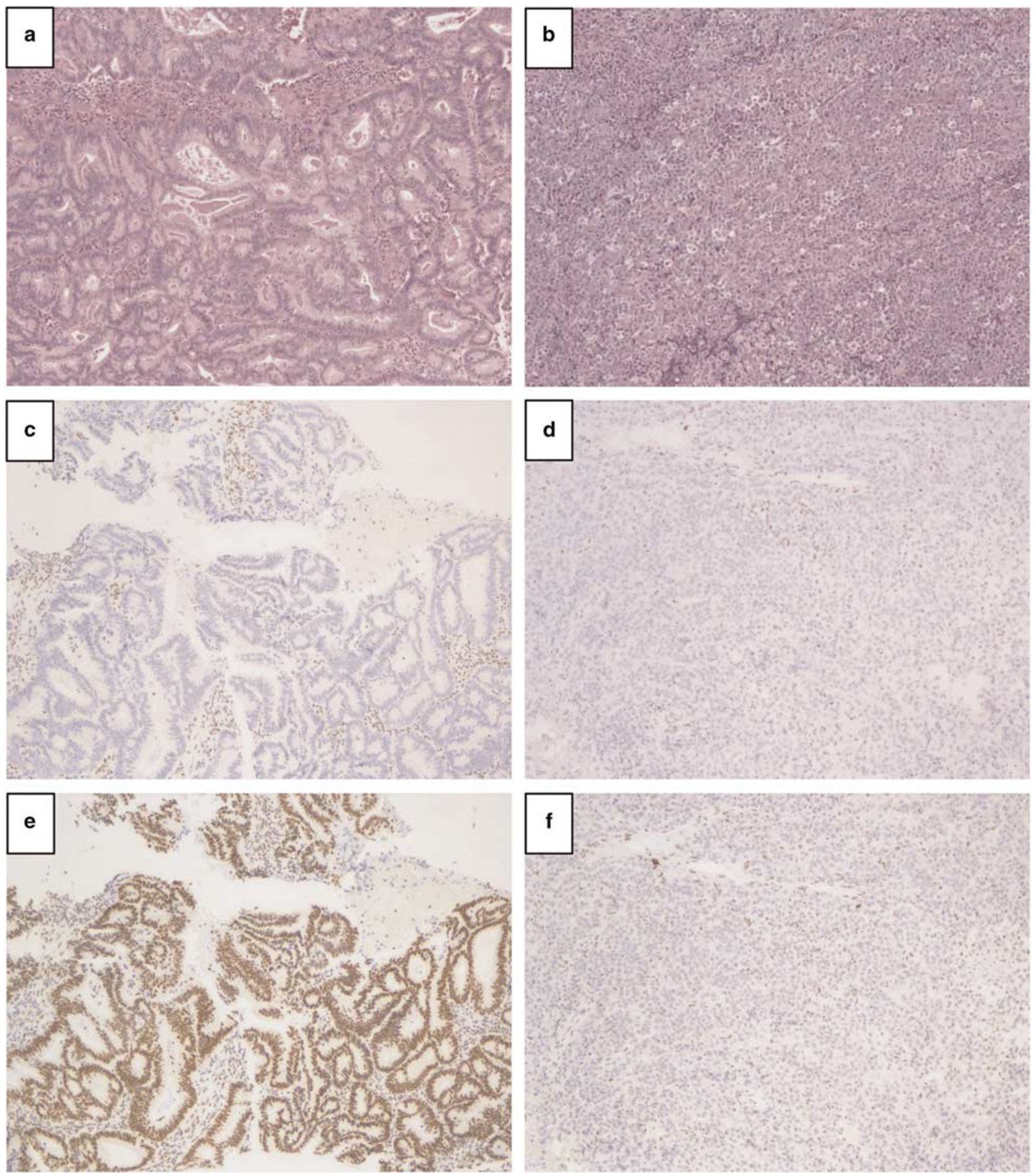

Figure 2 Concurrent absence of ARID1A and ARID1B expression in ARID1A/ARID1B co-mutated dedifferentiated carcinoma. The ARID1A and ARID1B immunostaining results of a representative case of ARID1A/ARID1B co-mutated dedifferentiated endometrial carcinoma (case 25, harbouring frameshift mutations involving both ARID1A and ARID1B) are illustrated. This tumour contained lowgrade endometrioid (a) and undifferentiated carcinoma components (b). ARID1A expression was absent in the tumour cells in both the endometrioid (c) and the undifferentiated components (d). ARID1B expression was present/intact in the endometrioid component (e) but was absent in the undifferentiated tumour component (f). The background stromal cells showed intact expression of ARID1A and ARID1B. 
deficiency by immunohistochemistry (7 with concurrent MLH1 and PMS2 loss and 2 with concurrent MSH2 and MSH6 loss) (Table 1). ${ }^{3,8}$

Clinically, patients with ARID1A/ARID1B co-inactivated dedifferentiated tumours ranged from 43 to 81 years in age (median age of 57 years), and $30 \%$ of the patients with ARID1A/ARID1B co-inactivated endometrial tumours presented with advanced stage (FIGO stages 3-4) disease. ARID1A/ ARID1B co-inactivated endometrial tumours and BRG1/INI1-inactivated endometrial tumours appear to exhibit similarly aggressive clinical behaviour to as $>50 \%$ of the patients in both groups died of their disease within 2 years of initial diagnosis $(P=0.15)$ (Figure 3). Histologically, there were no apparent differences in tumour morphology between ARID1A/ ARID1B co-inactivated tumours and BRG1 or INI1-inactivated tumours, as the inactivation of these SWI/SNF proteins both coincided with an undifferentiated histology. As previously characterized, ${ }^{12}$ the undifferentiated component of BRG1 or INI1inactivated tumours consistently demonstrated absent PAX8 and ER expression, whereas the undifferentiated component in 10 of 12 ARID1A/ ARID1B co-inactivated tumours showed absent PAX8 and ER expression. Two ARID1A/ARID1B co-inactivated tumours showed focally retained PAX8 expression and absent ER expression in the undifferentiated component.

\section{Discussion}

We identified in this study concurrent ARID1A/ ARID1B inactivating mutations that result in the loss

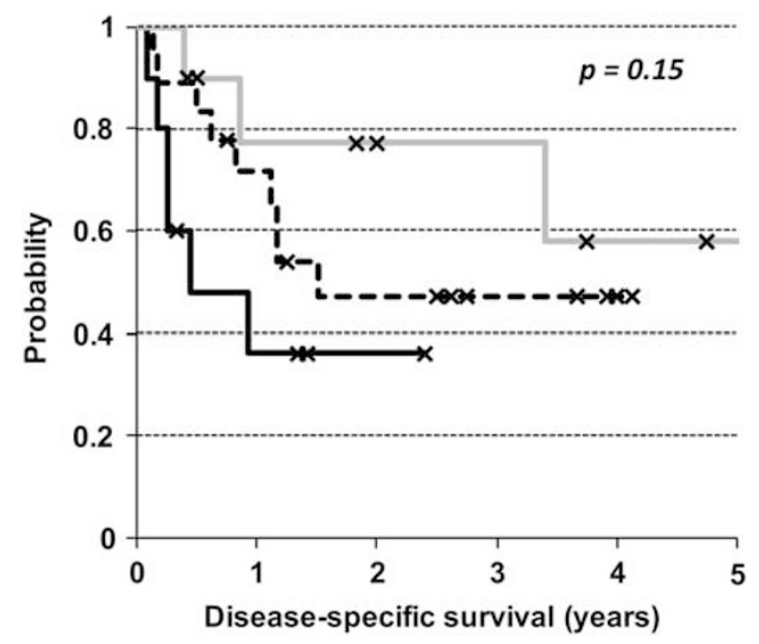

Figure 3 Kaplan-Meier disease-specific survival analysis comparing the different genetic subgroups of dedifferentiated endometrial carcinoma. ARID1A/ARID1B co-inactivated dedifferentiated endometrial carcinomas showed similarly aggressive clinical behaviour compared with SMARCA4-inactivated or SMARCAB1inactivated dedifferentiated endometrial carcinomas $(P=0.15)$ (solid black line: ARID1A/ ARID1B co-inactivated tumours; dotted black: SMARCA4 or SMARCB1-inactivated tumours; solid grey line: tumours with intact expression for SMARCA4/SMARCB1/ ARID1A/ARID1B). of these constitutively expressed proteins in the undifferentiated components of about a quarter of dedifferentiated endometrial/ovarian carcinoma. Together with the previously reported BRG1 inactivation and INI1 inactivation, inactivation of SWI/ SNF complex proteins is found in about $70 \%$ of dedifferentiated endometrial carcinomas. Moreover, the findings of ARID1A/ARID1B co-inactivation or BRG1 inactivation in the three dedifferentiated ovarian carcinomas examined suggest that ovarian endometrioid carcinomas can dedifferentiate through analogous mechanisms as seen in endometrial endometrioid carcinomas.

Inactivating $A R I D 1 A$ mutations are found in a wide range of cancers, and in the gynaecologic tract they are most frequently seen in endometriosisassociated ovarian neoplasms and in endometrioidtype endometrial carcinoma. ${ }^{18,19}$ In comparison, $A R I D 1 B$ mutations are less prevalent. ARID1A and ARID1B are alternate but obligatory DNA-binding subunits of the SWI/SNF-A complex, one of the mammalian SWI/SNF chromatin remodelling complexes that also include the SWI/SNF-B complex. ${ }^{18,19}$ Concurrent inactivating mutations that result in loss of both ARID1A and ARID1B are expected to abrogate the chromatin remodelling function of the SWI/SNF-A complex as it would lack the ability to bind and target DNA. The SWI/ SNF-B complex that requires a different set of DNA-binding subunits (ARID2, PBRM1 and BRD7) would in theory be functional in ARID1A/ARID1B co-inactivated tumours. In the context of dedifferentiated endometrial and ovarian carcinomas, ARID1A/ARID1B co-inactivation, BRG1 inactivation and INI1 inactivation appear to represent mutually exclusive contributing mechanisms for dedifferentiation, as the inactivation of these SWI/SNF complex proteins is associated with a loss of cellular differentiation resulting in an undifferentiated histology/immunophenotype concomitant with an aggressive clinical behaviour.

Helming et $a l^{20}$ recently showed that shRNAmediated depletion of ARID1B suppressed the in vitro growth and colony formation ability of ARID1A-deficient ovarian cancer cell lines and postulated the presence of synthetic lethality for ARID1B inactivation in ARID1A-mutated cancer. Although this appears to be at odds with our present observation, it is important to note that synthetic lethality is not the absolute phenomenon as the background cellular/molecular context is also an important determinant. ${ }^{21}$ Along with others, we have also published similar findings on BRG1/BRM dual deficiency where the in vitro data were in apparent disagreement with the clinical observation. ${ }^{3,22-25}$ The molecular and cellular contexts may not be equitable when one compares what appears to be synthetically lethal over a very short timeframe in an in vitro experimental system versus what eventually evolves over a much longer timeframe in human cancer. The findings presented here clearly 
demonstrated (in the selected scenario of dedifferentiated endometrial/ovarian carcinomas) that ARID1A and ARID1B co-deficiency does not impair the ability of the tumours to grow and disseminate in patients.

Our findings also showed that there is a greater propensity for MMR protein-deficient endometrioid carcinomas to give rise to ARID1A/ARID1B co-deficient undifferentiated tumour compared with MMR protein-proficient endometrioid carcinomas as $75 \%$ of ARID1A/ARID1B co-deficient dedifferentiated carcinomas are MMR protein deficient, and this is similar to what we observed with BRG1 or INI1-inactivated dedifferentiated carcinomas where $73 \%$ of these tumours are MMR protein deficient. ${ }^{3}$ This association between ARID1A/ARID1B co-inactivation and MMR protein deficiency is intriguing given that the only other two tumour types that have been documented to harbour recurrent ARID1A/ARID1B co-inactivating mutations, namely right-sided colonic adenocarcinoma and gastric adenocarcinoma (COSMIC database), were also likely MMR protein-deficient/ microsatellite-unstable tumours. ${ }^{26,27}$ Among the ARID1A/ARID1B co-mutated samples were three colon cancer cell lines-GP2D, SW48 and LIM2405, and all were microsatellite-instability high (MSI-H). Notably, in endometrial and ovarian dedifferentiated carcinomas, ARID1A loss appears to precede ARID1B loss. It is plausible that ARID1A inactivation in a MMR protein-deficient/MSI-H background more readily facilitates the acquisition of additional and necessary molecular aberrations to enable the successful emergence of an ARID1A/ ARID1B co-deficient undifferentiated sub-clone, compared with ARID1A inactivation in a MMR protein-proficient (microsatellite-stable) background. This postulate, if true, may help to reconcile the present finding of clinically aggressive ARID1A/ ARID1B co-deficient undifferentiated tumours with the prior observation that the knockdown of ARID1B suppresses the in vitro tumourigenic activity of ARID1A-deficient ovarian cancer cell lines. ${ }^{20}$ Further functional studies are clearly needed to determine if and how ARID1A and ARID1B co-deficiency contribute to cellular dedifferentiation that underlies the more aggressive clinical behaviour, and to determine whether additional corroborating molecular alterations are needed in both MMR protein-deficient and MMR protein-proficient settings.

In summary, we identified concurrent ARID1A and $A R I D 1 B$ inactivating mutations with consequent loss of protein expression in the undifferentiated component of approximately one-quarter of dedifferentiated endometrial and ovarian carcinomas. ARID1A and ARID1B co-inactivation appears to represent a major alternate mechanism to BRG1 or INI1 inactivation in the progression from endometrioid to undifferentiated carcinoma. These findings add to the multitude of mechanisms in which altered SWI/SNF complex activity may contribute to the development and progression of human cancers.

\section{Acknowledgments}

This study is supported by research funds from Cancer Research Society of Canada (20313), Royal Alexandra Hospital foundation, Women and Children's Health Research Institute, Alberta Cancer Foundation, Calgary Laboratory Services Internal Research Competition (RS10-536) and Canadian Cancer Society Research Institute-Innovation Grant (703458). Research reported in this publication was supported in part by the Cancer Center Support Grant of the National Institutes of Health/National Cancer Institute under award number P30CA008748.

\section{Disclosure/conflict of interest}

The authors declare no conflict of interest.

\section{References}

1 Silva EG, Deavers MT, Bodurka DC, et al. Association of low-grade endometrioid carcinoma of the uterus and ovary with undifferentiated carcinoma: a new type of dedifferentiated carcinoma? Int J Gynecol Pathol 2006;25:52-58.

2 Tafe LJ, Garg K, Chew I, et al. Endometrial and ovarian carcinomas with undifferentiated components: clinically aggressive and frequently underrecognized neoplasms. Mod Pathol 2010;23:781-789.

3 Karnezis AN, Hoang LN, Coatham M, et al. Loss of switch/sucrose non-fermenting complex protein expression is associated with dedifferentiation in endometrial carcinomas. Mod Pathol 2016;29:302-314.

4 Kuhn E, Ayhan A, Bahadirli-Talbott A, et al. Molecular characterization of undifferentiated carcinoma associated with endometrioid carcinoma. Am J Surg Pathol 2014;38:660-665.

5 Berretta R, Patrelli TS, Faioli R, et al. Dedifferentiated endometrial cancer: an atypical case diagnosed from cerebellar and adrenal metastasis: case presentation and review of literature. Int J Clin Exp Pathol 2013;6: 1652-1657.

6 Vita G, Borgia L, Di Giovannantonio L, et al. Dedifferentiated endometrioid adenocarcinoma of the uterus: a clinicopathologic study of a case. Int J Surg Pathol 2011;19:649-652.

7 Strehl JD, Wachter DL, Fiedler J, et al. Pattern of SMARCB1 (INI1) and SMARCA4 (BRG1) in poorly differentiated endometrioid adenocarcinoma of the uterus: analysis of a series with emphasis on a novel SMARCA4-deficient dedifferentiated rhabdoid variant. Ann Diagn Pathol 2015;19:198-202.

8 Stewart CJ, Crook ML. SWI/SNF complex deficiency and mismatch repair protein expression in undifferentiated and dedifferentiated endometrial carcinoma. Pathology 2015;47:439-445.

9 Ramalingam P, Masand RP, Euscher ED, et al. Undifferentiated carcinoma of the endometrium: an expanded immunohistochemical analysis including PAX-8 and basal-like carcinoma surrogate markers. Int J Gynecol Pathol 2016;35:410-418.

$10 \mathrm{Li} \mathrm{Z,} \mathrm{Zhao} \mathrm{C.} \mathrm{Clinicopathologic} \mathrm{and} \mathrm{immuno-}$ histochemical characterization of dedifferentiated 
endometrioid adenocarcinoma. Appl Immunohistochem Mol Morphol 2015; e-pub ahead of print.

11 Chen L, Pang S, Shen Y, et al. Low-grade endometrioid carcinoma of the ovary associated with undifferentiated carcinoma: case report and review of the literature. Int J Clin Exp Pathol 2014;7:4422-4427.

12 Hoang LN, Lee YS, Karnezis AN, et al. Immunophenotypic features of dedifferentiated endometrial carcinoma Insights from BRG1/INI1-deficient tumors. Histopathology 2016; doi:10.1111/his.12989; e-pub ahead of print.

13 Silva EG, Deavers MT, Malpica A. Undifferentiated carcinoma of the endometrium: a review. Pathology 2007;39:134-138.

14 Kobel M, Meng B, Hoang LN, et al. Molecular analysis of mixed endometrial carcinomas shows clonality in most cases. Am J Surg Pathol 2016;40:166-180.

15 Hoang LN, McConechy MK, Meng B, et al. Targeted mutation analysis of endometrial clear cell carcinoma. Histopathology 2015;66:664-674.

16 Hoang LN, Han G, McConechy M, et al. Immunohistochemical characterization of prototypical endometrial clear cell carcinoma-diagnostic utility of HNF-1beta and oestrogen receptor. Histopathology 2014;64:585-596.

17 Forbes SA, Beare D, Gunasekaran P, et al. COSMIC: exploring the world's knowledge of somatic mutations in human cancer. Nucleic Acids Res 2014;43:D805-D811.

18 Kadoch C, Crabtree GR. Mammalian SWI/SNF chromatin remodeling complexes and cancer: mechanistic insights gained from human genomics. Sci Adv 2015;1: e1500447.
19 Wilson BG, Roberts CW. SWI/SNF nucleosome remodellers and cancer. Nat Rev Cancer 2011;11:481-492.

20 Helming KC, Wang X, Wilson BG, et al. ARID1B is a specific vulnerability in ARID1A-mutant cancers. Nat Med 2014;20:251-254.

21 Chan N, Bristow RG. "Contextual" synthetic lethality and/or loss of heterozygosity: tumor hypoxia and modification of DNA repair. Clin Cancer Res 2010;16:4553-4560.

22 Hoffman GR, Rahal R, Buxton F, et al. Functional epigenetics approach identifies BRM/SMARCA2 as a critical synthetic lethal target in BRG1-deficient cancers. Proc Natl Acad Sci USA 2014;111:3128-3133.

23 Oike $\mathrm{T}$, Ogiwara $\mathrm{H}$, Tominaga $\mathrm{Y}$, et al. A synthetic lethality-based strategy to treat cancers harboring a genetic deficiency in the chromatin remodeling factor BRG1. Cancer Res 2013;73:5508-5518.

24 Reisman DN, Sciarrotta J, Wang W, et al. Loss of BRG1/BRM in human lung cancer cell lines and primary lung cancers: correlation with poor prognosis. Cancer Res 2003;63:560-566.

25 Karnezis AN, Wang Y, Ramos P, et al. Dual loss of the SWI/SNF complex ATPases SMARCA4/BRG1 and SMARCA2/BRM is highly sensitive and specific for small cell carcinoma of the ovary, hypercalcemic type. J Pathol 2016;238:389-400.

26 Jones S, Li M, Parsons DW, et al. Somatic mutations in the chromatin remodeling gene ARID1A occur in several tumor types. Hum Mutat 2012;33:100-103.

27 Wang K, Kan J, Yuen ST, et al. Exome sequencing identifies frequent mutation of ARID1A in molecular subtypes of gastric cancer. Nat Genet 2011;43: 1219-1223.

Supplementary Information accompanies the paper on Modern Pathology website (http://www.nature.com/ modpathol) 